\title{
EVALUATING THE COMPETITIVENESS OF QINZHOU PORT ON THAILAND- GUANGXI ROUTE UNDER PAN-BEIBU GULF ECONOMIC COOPERATION
}

\author{
Liurong Qin ${ }^{\text {, }}$ Boonsub Panichakarn ${ }^{\mathrm{b}}$ \\ ${ }^{a b}$ Naresuan University, Phitsanulok, Thailand \\ Corresponding email: qinliurong520@gmail.com
}

\begin{abstract}
This paper aims to, through Data Envelopment Analysis (DEA), analyze the efficiency and competitive position of the Qinzhou Port, compared with the rest eight ports of containerization in Pan-Beibu Gulf Economic Cooperation (PBGEC) region. Based on the introduction, a literature review is presented thereafter. We innovatively adopt the Charnes, Cooper and Rhodes (CCR) model, Banker, Charnes and Cooper (BCC) model to determine the overall efficiency, pure technical and scale efficiency, and then the Super Efficiency model has been used to indicate the efficiency ranking of the 9 ports in the PBGEC. The result implies that the Qinzhou port exists input redundancy and insufficient output due to its scale efficiency. Also, Qinzhou port is posed in an inferior position in its competitiveness. Further research is needed for how to overcome such weakness and then enhance the overall efficiency and competitiveness accordingly. At the end of this paper, limitations and recommendations are also presented.
\end{abstract}

Keywords: Qinzhou Port, Pan-Beibu Gulf Economic Cooperation (PBGEC), Data Envelopment Analysis.

\section{Introduction}

As a new sub-regional cooperation project within the frame of China- Association of Southeast Asian Nations (ASEAN) free trade zone, Pan-Beibu Gulf Economic Cooperation (PBGEC)has been posed as a hot issue for both China and ASEAN. As an initiative to create the costal industrial areas making full advantages in maritime transport with neighboring countries, China takes Qinzhou port as one of gateways for Thailand and Guangxi.

The evaluation of the competitiveness of Qinzhou port is critically important. From a nation's perspective, maritime transport becomes very important to the nation's integration. Over 90\% of international trade is through transporting by sea, and over 60\% China-ASEAN trade is through port logistics (Zou, 2012). In order to support trade oriented economic development, seaports have to improve port competitiveness by ensuring that seaport services are provided on an internationally competitive basis (Tongzon, 2001). Thus, a seaport with strong competitiveness is an important factor to a nation's international competitiveness. While, in terms of the significance of sea ports, Qinzhou Port, in particular, is the only foreign trade container port in Guangxi, the container hub in Beibu Gulf. From October, 2015, all of foreign trade container routes of other ports of Guangxi are transferred to the Qinzhou port except fruit routes. Bulk in Fangcheng port and Beihai port of Guangxi. In the past of 2015, the throughput of Qinzhou port reached 6.51 million tons, of which the throughput of container is 942,000 TEUs. So, Qinzhou port is the busiest container port in Guangxi (Qinzhou Municipal Bureau of Statistics, 2016), which paves the way for the Beibu Gulf port into an international shipping hub in Southeast Asia. 
However, evaluation of the container port competitiveness is not an easy case. Competitiveness is rather flexible and can be used for numerous different purposes in basic economic units (company, sector, region, country, and macro-region) (Vuković, 2012). In order to evaluate the seaport competitiveness, one of the most important tools to measure seaport competitiveness is the efficiency (Voorde and Winkelmans, 2001; Cruz, 2012).Therefore, this paperis to analyze the competitiveness of Qinzhou port in Data Envelopment Analysis (DEA) method through efficiency. For such purpose, what are to be compared and analyzed are Qiangzhou port and the rest 8 ports, namely, Hong Kong port, Thailand's Laem Chabang Port, Guangdong's Guangzhou port, Shenzhen port, Zhanjiang port, Zhuhai port, Hainan's Haikou port and Yangpu port.

The paper includes 5 sections. After the introduction, section 2 presents the review on the assessment method applied in port competitiveness. Section 3 explains the DEA method. Following section 4 are the data and result analysis of Qinzhou port competitiveness under DEA method. And section 5 summarizes the main result accordingly.

\section{Literature Review}

A variety of methods have been applied to evaluate port competitiveness. These studies can also be categorized into those that utilize subjective evaluation method and objective evaluation method. The former carries evaluation based on learned and experienced of human being to determine the weight of the index, and the latter confirms the weight of the index based on information and importance provided by index data itself.

Subjective evaluation method involves in Delphi method (Jafari et al., 2013), fuzzy comprehensive evaluation method and analytic hierarchy process (Yeo and Song, 2003; Song and Yeo, 2004; Yuen et al.,2016; Rudjanakanoknad et al., 2014; van Dyck and Ismael, 2015). Such method has the advantages of good applicability, operability and simplicity. However, its result may go far away from subjectivity since people were involved in the process of determining appraisal index and weight and influenced by the evaluator's cognitive ability and literacy levels.

Objective evaluation method includes DEA (Cullinane and Wang, 2010), factor analysis method (Yeo et al., 2008; Grosso and Monteiro, 2009) and principal component analysis (Tongzon and Heng, 2005). The objective evaluation method gives the weight of index objectively based on the information and importance provided by index data, which prevents the subjective effect from the evaluator.

Moreover, there have been many empirical studies of measuring port efficiency by using DEA, a simple and feasible scientific method suitable for evaluating port competitiveness through avoiding subjectivity and arbitrariness. Generally, DEA is a method of linear programming which uses the input, and output of productive processes in order to calculate the relative efficiency of each DMU (Polyzos and Niavis, 2013). Table 1 summaries the DEA method used in ports in recent 10 year. Most of the previous studies measured efficiency based on CCR model and BCC model. In this study, we are to use the CCR and BCC model to determine overall efficiency, pure technical, scale efficiency and adopt the Super Efficiency model to give the efficiency ranking of the 9 ports.

Table 1: Summary of the DEA Method Used in Ports

\begin{tabular}{|c|c|c|c|c|c|}
\hline Author (year) & DMU & Method & Input val & riables & Output variables \\
\hline $\begin{array}{l}\text { Al-Eraqi et al. } \\
(2008)\end{array}$ & $\begin{array}{l}22 \text { seaports in } \\
\text { the middle east } \\
\text { and east } \\
\text { African region }\end{array}$ & $\begin{array}{l}\text { CCR, } \\
\text { BCC }\end{array}$ & $\begin{array}{l}\text { Berth } \\
\text { storage } \\
\text { handling } \\
\text { equipment }\end{array}$ & $\begin{array}{r}\text { length, } \\
\text { area, }\end{array}$ & Throughput (tons) \\
\hline Liu et al. (2008) & $\begin{array}{l}45 \text { container } \\
\text { terminals in } \\
\text { mainland }\end{array}$ & BCC & $\begin{array}{l}\text { Quay } \\
\text { quayside } \\
\text { crane, }\end{array}$ & $\begin{array}{l}\text { length, } \\
\text { gantry } \\
\text { rubber- }\end{array}$ & Throughput (TEU) \\
\hline
\end{tabular}




\begin{tabular}{|c|c|c|c|c|}
\hline & China & & tyred gantry crane & \\
\hline $\begin{array}{l}\text { Min and Park } \\
(2008)\end{array}$ & $\begin{array}{l}11 \quad \text { container } \\
\text { terminals }\end{array}$ & $\mathrm{BCC}$ & $\begin{array}{l}\text { Gantry cranes, } \\
\text { terminal quay } \\
\text { length, yard areas, } \\
\text { size of labor force }\end{array}$ & $\begin{array}{l}\text { Throughput } \\
\text { (TEU), terminal } \\
\text { capacity (TEU) }\end{array}$ \\
\hline Wu and $\operatorname{Lin}(2008)$ & $\begin{array}{l}21 \text { leading } \\
\text { ports from G7, } \\
\text { BRIC and N-11 } \\
\text { countries }\end{array}$ & $\begin{array}{l}\text { CCR, } \\
\text { BCC }\end{array}$ & $\begin{array}{l}\text { Terminal area, } \\
\text { total quay length, } \\
\text { number of } \\
\text { quayside gantries, } \\
\text { number } \\
\text { straddle carries }\end{array}$ & $\begin{array}{l}\text { Number } \\
\text { containers }\end{array}$ \\
\hline Jiang and Li (2009) & $\begin{array}{lr}12 & \text { seaports } \\
\text { from } & \text { China, } \\
\text { Korea } & \text { and } \\
\text { Japan } & \end{array}$ & DEA & $\begin{array}{l}\text { Import/export by } \\
\text { customs, GDP by } \\
\text { regions, berth } \\
\text { length, } \\
\text { numbers }\end{array}$ & Throughput (TEU) \\
\hline $\begin{array}{l}\text { Sharma and Yu } \\
(2009)\end{array}$ & $\begin{array}{l}70 \text { container } \\
\text { terminals }\end{array}$ & DEA & $\begin{array}{l}\text { quay length (total } \\
\text { quay length of a } \\
\text { container } \\
\text { terminal), } \\
\text { terminal area , } \\
\text { quay } \\
\text { cranes,transfer } \\
\text { cranes(yard } \\
\text { cranes), straddle } \\
\text { carriers, reach } \\
\text { stackers }\end{array}$ & $\begin{array}{l}\text { Container } \\
\text { throughput }\end{array}$ \\
\hline $\begin{array}{l}\text { Wu and Goh } \\
\text { (2010) }\end{array}$ & $\begin{array}{l}20 \quad \text { largest } \\
\text { container ports }\end{array}$ & $\begin{array}{l}\text { CCR, } \\
\text { BCC }\end{array}$ & $\begin{array}{l}\text { Terminal area, } \\
\text { total quay length, } \\
\text { number of pieces } \\
\text { of equipment }\end{array}$ & $\begin{array}{l}\text { Container } \\
\text { throughput }\end{array}$ \\
\hline Gao et al. (2010) & & DEA & $\begin{array}{l}\text { Quay berth } \\
\text { number, } \\
\text { warehouse area, } \\
\text { storage yard area, } \\
\text { port aggregate } \\
\text { investment, cargo } \\
\text { gear number, } \\
\text { traveling bridge } \\
\text { number }\end{array}$ & $\begin{array}{l}\text { Container } \\
\text { throughput, cargo } \\
\text { throughput }\end{array}$ \\
\hline $\begin{array}{l}\text { Cullinane and } \\
\text { Wang (2010) }\end{array}$ & $\begin{array}{l}25 \text { leading } \\
\text { container ports }\end{array}$ & DEA & $\begin{array}{l}\text { Quayside gantry } \\
\text { (number), yard } \\
\text { gantry (number), } \\
\text { straddle carrier } \\
\text { (number) }\end{array}$ & $\begin{array}{l}\text { Container } \\
\text { throughput, } \\
\text { terminal length, } \\
\text { terminal area }\end{array}$ \\
\hline Eraqi et al. (2010) & $\begin{array}{l}22 \text { cargo } \\
\text { seaports in the } \\
\text { region of east } \\
\text { Africa and } \\
\text { middle east }\end{array}$ & $\begin{array}{c}\text { BCC, } \\
\text { CCR, } \\
\text { Super } \\
\text { efficiency }\end{array}$ & $\begin{array}{l}\text { Berth length, } \\
\text { terminal area, } \\
\text { equipment units }\end{array}$ & $\begin{array}{l}\text { Ships call, cargo } \\
\text { throughput (tons) }\end{array}$ \\
\hline Nigra (2010) & $\begin{array}{l}57 \text { worldwide } \\
\text { seaports (of } \\
\text { which } 21 \\
\text { Iberian portso }\end{array}$ & $\begin{array}{c}\text { BCC, } \\
\text { Super } \\
\text { efficiency }\end{array}$ & $\begin{array}{l}\text { Capital expense, } \\
\text { employees, } \\
\text { operational } \\
\text { expenses }\end{array}$ & $\begin{array}{l}\text { General cargo, dry } \\
\text { bulks/solid bulks, } \\
\text { liquid bulks, } \\
\text { passengers }\end{array}$ \\
\hline
\end{tabular}




\begin{tabular}{|c|c|c|c|c|}
\hline $\begin{array}{l}\text { Kamble et al. } \\
(2010)\end{array}$ & $\begin{array}{l}12 \text { major } \\
\text { Indian seaports }\end{array}$ & $\mathrm{BCC}$ & $\begin{array}{l}\text { Storage facilities, } \\
\text { number of berths, } \\
\text { number of cargo } \\
\text { handling } \\
\text { equipments }\end{array}$ & $\begin{array}{l}\text { Average total } \\
\text { turnaround time } \\
\text { (days), average } \\
\text { output per ship } \\
\text { berth day (tons) }\end{array}$ \\
\hline Wu et al. (2010) & $\begin{array}{l}77 \text { terminals } \\
\text { from } 56 \text { global } \\
\text { container ports }\end{array}$ & $\begin{array}{l}\text { CCR, } \\
\text { BCC }\end{array}$ & $\begin{array}{l}\text { Capacity of cargo } \\
\text { handling } \\
\text { equipment, } \\
\text { number of berths, } \\
\text { terminal area, } \\
\text { storage capacity }\end{array}$ & $\begin{array}{l}\text { Container } \\
\text { throughput }\end{array}$ \\
\hline Hung et al. (2011) & $\begin{array}{l}31 \text { container } \\
\text { ports in Asia- } \\
\text { Pacific region }\end{array}$ & $\begin{array}{l}\text { BCC, } \\
\text { CCR }\end{array}$ & $\begin{array}{l}\text { Terminal area, } \\
\text { ship-shore } \\
\text { container gantry } \\
\text { crane, container } \\
\text { berth, terminal } \\
\text { length (the length } \\
\text { of berths at which } \\
\text { container ships } \\
\text { anchor) }\end{array}$ & $\begin{array}{l}\text { Container } \\
\text { throughput }\end{array}$ \\
\hline $\begin{array}{l}\text { Munisamy and } \\
\text { Singh (2011) }\end{array}$ & $\begin{array}{l}69 \text { major Asian } \\
\text { container ports }\end{array}$ & $\begin{array}{l}\text { CCR, } \\
\text { BCC, } \\
\text { Super } \\
\text { efficiency }\end{array}$ & $\begin{array}{l}\text { Berth length, } \\
\text { terminal area, } \\
\text { total reefer points, } \\
\text { total quayside } \\
\text { cranes(and/or } \\
\text { Mobile cranes), } \\
\text { total yard } \\
\text { equipment }\end{array}$ & $\begin{array}{l}\text { Total throughput } \\
\text { (TEU) }\end{array}$ \\
\hline $\begin{array}{l}\text { Wanke et al. } \\
\text { (2011) }\end{array}$ & $\begin{array}{l}25 \text { major } \\
\text { Brazilian port } \\
\text { terminals }\end{array}$ & $\begin{array}{l}\text { CCR, } \\
\text { BCC }\end{array}$ & $\begin{array}{l}\text { Terminal area, size } \\
\text { of parking lot for } \\
\text { incoming } \\
\text { trucks/parking lot } \\
\text { (in number of } \\
\text { trucks), number of } \\
\text { shipping berths }\end{array}$ & $\begin{array}{l}\text { Aggregate } \\
\text { throughput per } \\
\text { year (in tons), } \\
\text { number of loaded } \\
\text { shipments per } \\
\text { year }\end{array}$ \\
\hline Zhang et al. (2011) & $\begin{array}{l}23 \text { container } \\
\text { terminals }\end{array}$ & DEA & $\begin{array}{l}\text { Number of berths, } \\
\text { berth length, land } \\
\text { size, quay crane, } \\
\text { yard gantries }\end{array}$ & Throughput (TEU) \\
\hline Demirel et al. (2012) & $\begin{array}{l}16 \text { container } \\
\text { terminals of } \\
\text { Mediterranean }\end{array}$ & $\begin{array}{l}\mathrm{BCC}, \\
\mathrm{CCR}\end{array}$ & $\begin{array}{l}\text { Quay length, } \\
\text { terminal area, } \\
\text { quay cranes } \\
\text { 9indclidng both } \\
\text { ship-to-shore and } \\
\text { the mobile quay } \\
\text { cranes used } \\
\text { mainly by small } \\
\text { container } \\
\text { terminals), yard } \\
\text { equipment, } \\
\text { maximum draft }\end{array}$ & Throughput (TEU) \\
\hline Kim (2012) & $\begin{array}{l}19 \text { European } \\
\text { container ports }\end{array}$ & DEA & $\begin{array}{l}\text { Length of berths, } \\
\text { terminal area, } \\
\text { number if cranes, } \\
\text { working hours }\end{array}$ & Throughput (TEU) \\
\hline
\end{tabular}




\begin{tabular}{|c|c|c|c|c|}
\hline $\begin{array}{l}\text { Azevedo et al. } \\
\text { (2012) }\end{array}$ & $\begin{array}{l}\text { 10 Iberian } \\
\text { container } \\
\text { terminals }\end{array}$ & $\mathrm{BCC}$ & $\begin{array}{l}\text { Container cranes, } \\
\text { terminal area, } \\
\text { quay length }\end{array}$ & $\begin{array}{l}\text { Container } \\
\text { throughput }\end{array}$ \\
\hline $\begin{array}{l}\text { Lu and Wang } \\
\text { (2012) }\end{array}$ & $\begin{array}{l}31 \text { major } \\
\text { container } \\
\text { terminals }\end{array}$ & $\begin{array}{c}\text { BCC, } \\
\text { CCR, } \\
\text { Super } \\
\text { efficiency }\end{array}$ & $\begin{array}{l}\text { Yard area, quay } \\
\text { crane, yard crane, } \\
\text { yard tractor, berth } \\
\text { length }\end{array}$ & $\begin{array}{l}\text { Container } \\
\text { throughput }\end{array}$ \\
\hline $\begin{array}{l}\text { Lu and Wang } \\
\text { (2012) }\end{array}$ & $\begin{array}{l}1 \text { container } \\
\text { terminals } \\
\text { (14China ports } \\
\text { an } 17 \text { Korea } \\
\text { ports) }\end{array}$ & $\begin{array}{l}\text { CCR, } \\
\text { BCC }\end{array}$ & $\begin{array}{l}\text { Yard area per } \\
\text { berth, number of } \\
\text { quayside cranes } \\
\text { per berth, number } \\
\text { of terminal cranes } \\
\text { per berth, number } \\
\text { of yard tractor per } \\
\text { berth, berth } \\
\text { length, water } \\
\text { depth }\end{array}$ & $\begin{array}{l}\text { Container } \\
\text { throughput per } \\
\text { berth }\end{array}$ \\
\hline $\begin{array}{l}\text { Niavis and } \\
\text { Tsekeris (2013) }\end{array}$ & $\begin{array}{l}\text { 30 seaports in } \\
\text { the wider } \\
\text { region of } \\
\text { south-eastern } \\
\text { Europe }\end{array}$ & $\begin{array}{c}\text { DEA, } \\
\text { Super } \\
\text { efficiency }\end{array}$ & $\begin{array}{l}\text { Number of berth, } \\
\text { length of quays, } \\
\text { number of cranes } \\
\text { used by each port } \\
\text { for container } \\
\text { handling }\end{array}$ & $\begin{array}{l}\text { Total throughput } \\
\text { (TEU) }\end{array}$ \\
\hline $\begin{array}{l}\text { Pjevcev et al. } \\
\text { (2012) }\end{array}$ & $\begin{array}{l}5 \text { Serbia river } \\
\text { ports of } \\
\text { Danube river }\end{array}$ & DEA & $\begin{array}{l}\text { Total area of } \\
\text { warehouses, quay } \\
\text { length, number of } \\
\text { cranes }\end{array}$ & $\begin{array}{l}\text { Port throughput } \\
\text { (tons) }\end{array}$ \\
\hline Bichou (2012) & $\begin{array}{l}420 \text { container } \\
\text { terminals }\end{array}$ & $\begin{array}{l}\text { CCR, } \\
\text { BCC }\end{array}$ & $\begin{array}{l}\text { Terminal area, } \\
\text { max draft, length } \\
\text { overall, quay crane } \\
\text { index, yard- } \\
\text { stacking }\end{array}$ & Throughput (TEU) \\
\hline $\begin{array}{l}\text { Infante and } \\
\text { Gutiérrez (2013) }\end{array}$ & $\begin{array}{l}33 \\
\text { ports/terminals } \\
\text { in the Asian } \\
\text { Pacific region }\end{array}$ & $\begin{array}{l}\text { CCR, } \\
\text { BCC }\end{array}$ & $\begin{array}{l}\text { Total number of } \\
\text { gantry, terminal } \\
\text { area, total berth } \\
\text { length of the } \\
\text { terminals }\end{array}$ & TEUs handled \\
\hline $\begin{array}{l}\text { Lu and Park } \\
(2013)\end{array}$ & $\begin{array}{l}28 \text { major East } \\
\text { Asia container } \\
\text { terminals }\end{array}$ & CCR & $\begin{array}{l}\text { Yard area, quay } \\
\text { crane, terminal } \\
\text { crane, yard } \\
\text { tractor, berth } \\
\text { length }\end{array}$ & Throughput (TEU) \\
\hline $\begin{array}{l}\text { Mokhtar and } \\
\text { Shah (2013) }\end{array}$ & $\begin{array}{l}6 \text { major } \\
\text { container } \\
\text { terminals in } \\
\text { Malaysia }\end{array}$ & $\begin{array}{l}\text { CCR, } \\
\text { BCC }\end{array}$ & $\begin{array}{l}\text { Total terminal } \\
\text { area, maximum } \\
\text { draft in meter, } \\
\text { berth length in } \\
\text { meter, quay crane } \\
\text { index, yard } \\
\text { stacking index, } \\
\text { vehicles, number } \\
\text { of gate lanes }\end{array}$ & Throughput (TEU) \\
\hline $\begin{array}{l}\text { Munisamy and } \\
\text { Jun (2013); } \\
\text { Munisamy and } \\
\text { Wang (2013) }\end{array}$ & $\begin{array}{l}69 \text { major } \\
\text { container ports } \\
\text { in Asia }\end{array}$ & DEA & $\begin{array}{l}\text { Berth length, } \\
\text { terminal area, } \\
\text { total reefer points, } \\
\text { total quayside }\end{array}$ & $\begin{array}{l}\text { Total throughput } \\
\text { (TEU) }\end{array}$ \\
\hline
\end{tabular}




\begin{tabular}{|c|c|c|c|c|}
\hline & & & $\begin{array}{l}\text { cranes, total yard } \\
\text { equipment }\end{array}$ & \\
\hline $\begin{array}{l}\text { Munisamy and } \\
\text { Jun (2013) }\end{array}$ & $\begin{array}{l}\text { 30 Latin } \\
\text { America } \\
\text { container } \\
\text { seaports }\end{array}$ & $\begin{array}{l}\text { BCC, } \\
\text { CCR }\end{array}$ & $\begin{array}{l}\text { Berth length, } \\
\text { terminal area, } \\
\text { quay equipment, } \\
\text { yard gantry, } \\
\text { sophisticated } \\
\text { equipment (reach } \\
\text { stackers, straddle } \\
\text { carriers), general } \\
\text { equipment } \\
\text { 9forklifts and yard } \\
\text { tractors) }\end{array}$ & $\begin{array}{l}\text { Container } \\
\text { throughput }\end{array}$ \\
\hline Grilo (2013) & $\begin{array}{l}11 \text { terminals of } \\
\text { Portuguese } \\
\text { ports }\end{array}$ & $\begin{array}{l}\text { CCR, } \\
\text { BCC }\end{array}$ & $\begin{array}{l}\text { Total quay length, } \\
\text { number of berths, } \\
\text { quay depth, } \\
\text { storage area, } \\
\text { number of cranes }\end{array}$ & $\begin{array}{l}\text { Cargo throughput } \\
\text { (ton) }\end{array}$ \\
\hline $\begin{array}{l}\text { Olapojuand } \\
\text { Aloba (2013) }\end{array}$ & Lagos seaports & DEA & $\begin{array}{l}\text { Terminal area, } \\
\text { berth number, } \\
\text { berth length }\end{array}$ & $\begin{array}{l}\text { Ship traffic, cargo } \\
\text { throughput }\end{array}$ \\
\hline $\begin{array}{l}\text { Polyzos and } \\
\text { Niavis (2013) }\end{array}$ & $\begin{array}{l}30 \\
\text { Mediterranean } \\
\text { ports }\end{array}$ & $\begin{array}{l}\text { CCR, } \\
\text { Super } \\
\text { efficiency }\end{array}$ & $\begin{array}{l}\text { Length of quays, } \\
\text { number of ship to } \\
\text { shore cranes }\end{array}$ & $\begin{array}{l}\text { Number of TEUs } \\
\text { that were moved }\end{array}$ \\
\hline $\begin{array}{l}\text { Shin and Jeon } \\
\text { (2013) }\end{array}$ & $\begin{array}{l}8 \text { terminals of } \\
\text { south Korea }\end{array}$ & $\begin{array}{l}\text { BCC, } \\
\text { CCR }\end{array}$ & $\begin{array}{l}\text { Quay length, } \\
\text { number of } \\
\text { container cranes, } \\
\text { area of container } \\
\text { yard }\end{array}$ & $\begin{array}{l}\text { Container } \\
\text { throughput and } \\
\mathrm{CO}_{2} \text { emission }\end{array}$ \\
\hline $\begin{array}{l}\text { SchøyenandOdeck } \\
\text { (2013) }\end{array}$ & $\begin{array}{l}24 \text { container } \\
\text { ports from } \\
\text { Norway, all } \\
\text { Nordic } \\
\text { countries, } \\
\text { United } \\
\text { Kingdom }\end{array}$ & $\begin{array}{l}\text { BCC, } \\
\text { CCR }\end{array}$ & $\begin{array}{l}\text { Berth length, } \\
\text { terminal area, } \\
\text { yard gantry } \\
\text { cranes, straddle } \\
\text { carriers }\end{array}$ & $\begin{array}{l}\text { Container } \\
\text { handling trucks, } \\
\text { container } \\
\text { throughput }\end{array}$ \\
\hline Yuen et al. (2013) & $\begin{array}{l}21 \text { major } \\
\text { container } \\
\text { terminals in } \\
\text { China, Busan, } \\
\text { Singapore and } \\
\text { Kaohsiung }\end{array}$ & DEA & $\begin{array}{l}\text { Number of berths, } \\
\text { total berth length, } \\
\text { land size (port } \\
\text { land area), } \\
\text { number of quay } \\
\text { cranes, yard } \\
\text { gantries }\end{array}$ & $\begin{array}{l}\text { Cargo throughput } \\
\text { (TEU) }\end{array}$ \\
\hline $\begin{array}{l}\text { Rajasekar et al. } \\
(2014)\end{array}$ & $\begin{array}{l}\text { Major ports in } \\
\text { India }\end{array}$ & $\begin{array}{l}\text { CCR, } \\
\text { BCC }\end{array}$ & $\begin{array}{l}\text { Number of berth, } \\
\text { berth length, } \\
\text { number of } \\
\text { equipments, } \\
\text { number of } \\
\text { employees }\end{array}$ & $\begin{array}{l}\text { Container } \\
\text { throughput )TEU), } \\
\text { total traffic }\end{array}$ \\
\hline Akgül et al. (2015) & $\begin{array}{l}15 \text { leading } \\
\text { container ports } \\
\text { in Turkey }\end{array}$ & CCR & $\begin{array}{l}\text { Number of quay } \\
\text { cranes, terminals } \\
\text { area, storage } \\
\text { capacity, quay } \\
\text { length }\end{array}$ & Throughput (TEU) \\
\hline Almawshe and Shah & 19 container & CCR & Terminal area, & Throughput (TEU) \\
\hline
\end{tabular}




\begin{tabular}{|c|c|c|c|c|}
\hline (2015) & $\begin{array}{l}\text { terminals in } \\
\text { the middle } \\
\text { eastern region }\end{array}$ & & $\begin{array}{l}\text { quay length, quay } \\
\text { crane, yard } \\
\text { equipment, } \\
\text { maximum draft }\end{array}$ & \\
\hline $\begin{array}{l}\text { Baran and } \\
\text { Górecka (2015) }\end{array}$ & $\begin{array}{l}18 \text { leading } \\
\text { container ports } \\
\text { ranked in } 2012\end{array}$ & $\begin{array}{l}\text { CCR, } \\
\text { BCC }\end{array}$ & $\begin{array}{l}\text { Number of berths, } \\
\text { terminal area, } \\
\text { storage capacity in } \\
\text { TEU, quay length }\end{array}$ & $\begin{array}{l}\text { Annual } \\
\text { throughput (TEU) }\end{array}$ \\
\hline Ding et al. (2015) & $\begin{array}{l}21 \text { coastal small } \\
\text { and medium } \\
\text { sized-port } \\
\text { container } \\
\text { terminals in } \\
\text { China }\end{array}$ & $\begin{array}{l}\text { CCR, } \\
\text { BCC }\end{array}$ & $\begin{array}{l}\text { Terminal length, } \\
\text { handling } \\
\text { equipment } \\
\text { quantity, staff } \\
\text { quantity }\end{array}$ & $\begin{array}{l}\text { Container } \\
\text { throughput }\end{array}$ \\
\hline Lu et al. (2015) & $\begin{array}{l}20 \text { world's } \\
\text { container ports }\end{array}$ & $\begin{array}{c}\text { CCR, } \\
\text { BCC, } \\
\text { Super } \\
\text { efficiency }\end{array}$ & $\begin{array}{l}\text { Yard area, quay } \\
\text { crane, terminal } \\
\text { crane, yard } \\
\text { tractor, berth } \\
\text { length }\end{array}$ & $\begin{array}{l}\text { Container } \\
\text { throughput }\end{array}$ \\
\hline $\begin{array}{l}\text { Nguyen et al. } \\
\text { (2015) }\end{array}$ & $\begin{array}{l}43 \text { largest } \\
\text { Vietnamese } \\
\text { ports }\end{array}$ & DEA & $\begin{array}{l}\text { Berth length, } \\
\text { terminal areas, } \\
\text { warehouse } \\
\text { capacity, cargo } \\
\text { handling } \\
\text { equipment }\end{array}$ & Cargo throughput \\
\hline $\begin{array}{l}\text { da Cruz and de } \\
\text { Matos Ferreira } \\
\text { (2016) }\end{array}$ & $\begin{array}{l}\text { 10 Iberian } \\
\text { seaport }\end{array}$ & $\begin{array}{l}\text { CCR, } \\
\text { BCC }\end{array}$ & $\begin{array}{l}\text { Labor, fixed asset, } \\
\text { turnover, ships } \\
\text { handled }\end{array}$ & Cargo throughput \\
\hline Jang et al. (2016) & $\begin{array}{l}21 \text { container } \\
\text { ports in Asia }\end{array}$ & $\begin{array}{l}\text { CCR, } \\
\text { BCC, } \\
\text { SBM }\end{array}$ & $\begin{array}{l}\text { Number of berth, } \\
\text { length of berth, } \\
\text { terminal area, } \\
\text { gantry crane }\end{array}$ & $\begin{array}{l}\text { Cargo volume } \\
\text { (TEU) }\end{array}$ \\
\hline $\begin{array}{l}\text { Tetteh et al. } \\
\text { (2016) }\end{array}$ & $\begin{array}{l}\text { China and } 5 \\
\text { west African } \\
\text { countries }\end{array}$ & DEA & $\begin{array}{l}\text { Number of berths, } \\
\text { number of cranes, } \\
\text { length of quay }\end{array}$ & $\begin{array}{l}\text { Vessel calls(gross } \\
\text { tons), port } \\
\text { throughput (TEU) }\end{array}$ \\
\hline $\begin{array}{l}\text { Zheng and Park } \\
\text { (2016) }\end{array}$ & $\begin{array}{l}30 \text { major } \\
\text { container } \\
\text { terminals in } \\
\text { Korea and } \\
\text { China }\end{array}$ & $\begin{array}{l}\text { CCR, } \\
\text { BCC }\end{array}$ & $\begin{array}{l}\text { Berth length, yard } \\
\text { area, number of } \\
\text { quay cranes, } \\
\text { number of yard } \\
\text { cranes }\end{array}$ & Throughput (TEU) \\
\hline $\begin{array}{l}\text { Schøyen and } \\
\text { Odeck (2017) }\end{array}$ & $\begin{array}{l}6 \text { largest } \\
\text { Norwegian } \\
\text { container ports } \\
\text { against } 14 \\
\text { similar small- } \\
\text { and medium- } \\
\text { sized ports in } \\
\text { the Nordic } \\
\text { countries and } \\
\text { UK }\end{array}$ & DEA & $\begin{array}{l}\text { Berth length, } \\
\text { terminal areas, } \\
\text { number of yard } \\
\text { gantrycranes, } \\
\text { straddle carries, } \\
\text { container handling } \\
\text { trucks }\end{array}$ & $\begin{array}{l}\text { Container units } \\
\text { (TEU) }\end{array}$ \\
\hline
\end{tabular}

Note: CCR: Charnes, Cooper and Rhodes model; BCC: Banker, Charnes and Cooper model; DEA: Data Envelopment Analysis; G7: America, France, Italy, Canada, Germany, Japan, and the England; BRIC: Brazil, Russia, India, China; N11: South Korea, Indonesia, Vietnam, the Philippines, Pakistan, Bangladesh in Asia, Nigeria and Egypt in Africa, Mexico in North America, Iran in the Middle East, and Turkey 
As indicated in the Table 1, there have been many empirical studies on measuring port efficiency by using DEA in the literature. The models mostly used to apply the DEA method are the CCR model and BCC model. However, it should be pointed out that few studies have been less investigated the competitiveness of Qinzhou port. In particular, the studies that combined with DEA method used in Qinzhou port cannot be found.

\section{Methodology}

\subsection{Data Envelopment Analysis}

DEA is a multi-factor productivity analysis for measuring the relative efficiencies on Decision Making Units (DMUs). In this study, the DEA-CCR and DEA-BBC models are to be adopted for analysis the relative efficiency of the sample ports, both input and output oriented. Because the above models are given a value of 1 for all efficiency DMUs, it is unable to establish any further distinctions among the efficiency DMUs. Therefore, the DEA-Super Efficiency model is to be used to rank the ports, and the new efficiency value can thus be greater than 1.

Assuming that there are n DMUs, where each $D M U_{j}(j=1, \cdots, n)$ produces s output $y_{r j}(r=$ $1, \cdots, s)$ by utilizing $m$ inputs $x_{i j}(i=1, \cdots, m)$.

Then it has a linear programming dual of CCR model as follows:

$$
\begin{aligned}
& \operatorname{Min} \theta-\varepsilon {\left[\sum_{r=1}^{s} s_{r}^{+}+\sum_{i=1}^{m} s_{i}^{-}\right] } \\
& \text {Subject to: } \sum_{j=1}^{n} x_{i j} \lambda_{j}+s_{i}^{-}=\theta x_{i j} \\
& \sum_{j=1}^{n} y_{r j} \lambda_{j}-s_{r}^{+}=y_{r j} \\
& \lambda_{j} \geq 0, s_{r}^{+}, s_{i}^{-} \geq \varepsilon \geq 0 .
\end{aligned}
$$

Where: $y_{r j}$ is $r$ th outputs of the $j$ th DMU;

$x_{i j}$ isith inputs of the $j$ th DMU;

$\varepsilon$ is a small positive number;

$\lambda_{j_{i}}$ is a weight of $j$ th DMU;

$S_{r}^{+}$is a slack variable of $r$ th output;

$s_{i}^{-}$is a slack variable of $i$ thinput.

The BCC model adds the convexity restriction $\left(\sum_{j=1}^{s} \lambda_{j}=1\right)$ based on formula (1). The linear programming dual of BCC model is represented by:

$$
\underset{\operatorname{Min}}{\theta} \theta-\varepsilon\left[\sum_{r=1}^{s} s_{r}^{+}+\sum_{r=1}^{m} s_{i}^{-}\right]
$$

$\prod_{\substack{\infty \\ 0 \\ 0}}^{N}$

Subject to: $\sum_{j=1}^{n} x_{i j} \lambda_{j}+s_{i}^{-}=\theta x_{i j}$ 


$$
\begin{aligned}
& \sum_{j=1}^{n} y_{r j} \lambda_{j}-s_{r}^{+}=y_{r j} \\
& \sum_{j=1}^{s} \lambda_{j}=1 \\
& \lambda_{j}, s_{r}^{+}, s_{i}^{-} \geq 0
\end{aligned}
$$

The Super Efficiency model is formulated in the following form similar to the above two models:

$$
\begin{aligned}
& \operatorname{Min} \boldsymbol{\theta} \\
& \text { Subject to: } \sum_{j=1, j \neq j_{0}}^{n} \lambda_{j} x_{j}+s_{i}^{-}=\theta x_{i j} \\
& \sum_{j=1, j \neq j_{0}}^{n} \lambda_{j} y_{j}-s_{r}^{+}=y_{r j} \\
& \lambda_{j}, s_{r}^{+}, s_{i}^{-} \geq 0
\end{aligned}
$$

\subsection{Input and Output Variables}

There have been many empirical studies on measuring port efficiency by using DEA in the literature. The input and output variables used in these studies are presented in Table 1.

To avoid having too many DMUs with efficiency values being equal to 1 , which would lower the discriminatory power of DEA, Norman and Stoker (1991) suggested that the number of DMUs should be at least twice the sum of input and output variables. Because the present study had nine ports, adopting this suggestion would mean that the sum of input and output variables could not be greater than four (Golany and Roll, 1989). Accordingly, the number of berth, berth length (meter), and terminal area (square meter) are considered as input variables, and container throughput (TEU) is used as the output variables in this study. The summary of input and output variables as shown in table 2.

Table 2: input and output variables used in the DEA model

\begin{tabular}{clc}
\hline Variables & \multicolumn{1}{c}{ Description } & Measurement unit \\
\hline Input: & $\begin{array}{l}\text { Total container berths in } \\
\text { container port }\end{array}$ & Number \\
Berth length & $\begin{array}{l}\text { Total container berth length of } \\
\text { container port }\end{array}$ & Meter \\
Terminal area & $\begin{array}{l}\text { total area of container terminals } \\
\text { in container port }\end{array}$ & Hectare \\
Output: & $\begin{array}{l}\text { Total container throughput in } \\
\text { TEU }\end{array}$ & 10, ooo TEU/year \\
\hline
\end{tabular}




\subsection{Data Collection}

Necessary information and data have been collected through precious literature, telephone interview, statistical yearbook, official websites from ports and the port authority and the other specialized websites.

Table3:Data for DEA Model for Year 2015

\begin{tabular}{ccccc}
\hline DMU: & $\begin{array}{c}\text { Input 1: } \\
\text { Number of } \\
\text { berths } \\
\text { (number) }\end{array}$ & $\begin{array}{c}\text { Input 2: } \\
\text { Berth length } \\
(\mathrm{m})\end{array}$ & $\begin{array}{c}\text { Input 3: } \\
\text { Terminal } \\
\text { area } \\
\text { (hectare) }\end{array}$ & $\begin{array}{c}\text { Output: } \\
\text { Container } \\
\text { throughput } \\
\text { (10,000 TEU) }\end{array}$ \\
\hline Qinzhou & 4 & 2300 & 158 & 94.2 \\
Shenzhen & 39 & 16943 & 792 & 2420.45 \\
Guangzhou & 68 & 13241 & 899.86 & 1762.49 \\
Zhanjiang & 2 & 678 & 67.8 & 60.12 \\
Zhuhai & 24 & 5078 & 228.6 & 133.77 \\
Haikou & 3 & 786 & 30 & 127.13 \\
Yangpu & 9 & 1696 & 23.1445 & 27.16 \\
Hong Kong & 24 & 7694 & 279 & 2011.4 \\
Laem Chabang & 11 & 4640 & 256.6757 & 679.4
\end{tabular}

Source: China Ports Year Book 2016; China Statistical Yearbook 2016; Port of Hong Kong in Figures 2015; BSAA Annual Report 16-17; telephone interview; office websites of government, terminal, port and port authority

\section{Results and Discussion}

In this study, both the CCR and BCC models are applied to the MaxDEA software. The super efficiency model isapplied to the EMS1.3 software.

\subsection{Input Oriented CCR Model}

Table 4shows the relative efficiency ranking of 9 ports under input oriented CCR model. Hong Kong port's efficiency performs the best (the score is 1), other ports efficiency value are less than 0.8, and Zhuhai port performs the worst (0.101). Such score of the Qinzhou port is 0.281 .

As the table shows, under the input oriented CCR model, Hong Kong port is efficiency (efficiency value $=1$ ). In the table, proportionate movement represents input redundancy value and slack movement indicates the insufficient output value and projection denotes the efficiency target value. The projection results provide a performance improvement target for inefficiency ports. That is, Qingzhou port should achieve 9420 thousand TEU with 1 berth, 360 meter berth length and 13 ha terminal area.

Hong Kong port relative efficiency value is 1, target and actual values are the same, and it has advantages in the PBGEC. In order to analyze the inefficiency port problem in terms of input, Table 5 makes a percentage difference in comparison with the target value and actual value (original data).

There is a large gap between the target value and original data. This shows that the efficiency of inputs being used is not appropriate, a serious input crowding in the port on the given level of input. A relatively large percentage difference in the ports except Shenzhen port, Laem Chabang port and Haikou port.The degree of input crowing is more than $25 \%$. Yangpu port is the highest crowding degree port with berth input crowding value is $96.4 \%$. It is obvious that degree of resource waste is serious. 


\subsection{Output Oriented CCR Model}

The relative efficiency ranking of input-oriented CCR and output-oriented CCR are the same, but output-oriented CCR gives the target value (projection) of output (container throughput). Therefore, it is necessary to show the calculating results of output-oriented CCR.

In the table 6, the results give the optimized resource allocation target value to ports, such as Qinzhou port should reduce 1017.667 meter berth length (input 2), 111.5 ha terminal area (input 3) to increase 24.1033 million TEU (proportionate movement value of output) and then achieve 335.233 thousand TEU (projection movement value of output). Other inefficiency port can be analyzed similarly. Also, the efficiency target value and original data are to be compared in Table 7. Apparently, the inefficiency ports can achieve a large output at the current level of input. For instance, Qinhzou port can realize DEA efficiency when it enlarges $71.9 \%$ container throughput at the current level.

Table 6: Relative Efficiency Calculation Results under Output Oriented CCR

\begin{tabular}{cccccc}
\hline Port & $\begin{array}{c}\text { Slack } \\
\text { movement } \\
\text { (Input 1) }\end{array}$ & $\begin{array}{c}\text { Slack } \\
\text { movement } \\
\text { (Input 2) }\end{array}$ & $\begin{array}{c}\text { Slack } \\
\text { movement } \\
\text { (input 3) }\end{array}$ & $\begin{array}{c}\text { Proportionat } \\
\text { e movement } \\
\text { (output) }\end{array}$ & $\begin{array}{c}\text { Projection } \\
\text { movement } \\
\text { (output) }\end{array}$ \\
\hline Hong Kong & o & 0 & 0 & 0 & 2011.4 \\
Shenzhen & 0 & -4440.25 & -338.625 & 848.075 & 3268.525 \\
Laem Chabang & 0 & -113.583 & -128.801 & 242.492 & 921.892 \\
Haikou & -0.548 & 0 & -1.498 & 78.35 & 205.48 \\
Guangzhou & -26.697 & 0 & -419.715 & 1699.032 & 3461.522 \\
Zhanjiang & 0 & -36.833 & -44.55 & 107.497 & 167.617 \\
Qinzhou & 0 & -1017.667 & -111.5 & 241.033 & 335.233 \\
Yangpu & -7.009 & -1057.743 & 0 & 139.696 & 166.856 \\
Zhuhai & -8.16 & 0 & -44.461 & 1193.744 & 1327.514 \\
\hline
\end{tabular}

Note: The proportionate movement of input and slack movement of the output value are 0 . Here will not be listed.

\subsection{Input Oriented BCC}

Through contrasting Table 4withTable 8, relative efficiency values under output-oriented BCC have a big change. There are 5 ports are DEA efficiency: Zhanjiang, Yangpu, Shenzhen, Hong Kong and Haikou ports. The inefficiency ports' relative efficiency values are generally higher, only Zhuhai port's relative efficiency value below 0.5 .

The relative efficiency result details of other inefficiency ports are summarized in the Table 8 . Proportionate movement and slack movement values are very small compared to the values of CCR model. Compared the port ranking of CCR model (consider constant returns to scale), Laem Chabang port and Guangzhou port drop in ranking under the BCC (variable returns to scale), we hold that these two ports are not achieving DEA efficiency the reason is the scale of ports is expanding rapidly but a good integration of resources is not done yet. On the other hand, the Laem Chabang port and Guangzhou port have a huge potential. 


\subsection{Output Oriented BCC}

Table 9 is the relative efficiency ranking under output-oriented BCC model. The relative efficiency scores with small gaps compared to the scores of input-oriented BCC. The relative efficiency value is between 0.1-0.8, Zhuhai port is the smallest one with the score 0.103.

To analyze the gap between the original data and the target value (projection value) which the port achieve DEA efficiency is required. It is found that there are large gaps between the original data and the target value. The target value of Qinzhou port is 2.5 times than the original value. The target value of Zhuhai port even up to 9.7 times. It is worthy of pointing out that if Guangzhou port achieves DEA efficiency, its output will have greatly increased, thus enhancing its competitiveness.

Table 9: Relative Efficiency Results under Output-Oriented BCC Model

\begin{tabular}{|c|c|c|c|c|c|c|c|}
\hline \multirow{2}{*}{ Port } & \multirow{2}{*}{$\begin{array}{c}\text { Scor } \\
\mathrm{e}\end{array}$} & Input 1 & Input 2 & Input 3 & \multicolumn{3}{|c|}{ Output } \\
\hline & & $\begin{array}{c}\text { Slack } \\
\text { movemen } \\
t\end{array}$ & $\begin{array}{c}\text { Slack } \\
\text { movemen } \\
\mathrm{t}\end{array}$ & $\begin{array}{c}\text { Slack } \\
\text { movemen } \\
t\end{array}$ & $\begin{array}{l}\text { Origina } \\
1\end{array}$ & $\begin{array}{l}\text { Proportionat } \\
\text { e movement }\end{array}$ & $\begin{array}{c}\text { Projectio } \\
n\end{array}$ \\
\hline Zhanjiang & 1 & 0 & O & O & 60.12 & $\mathrm{O}$ & 60.12 \\
\hline Yangpu & 1 & $\mathrm{O}$ & O & $\mathrm{O}$ & 27.16 & O & 27.16 \\
\hline Shenzhen & 1 & $\mathrm{O}$ & O & O & $\begin{array}{c}2420.4 \\
5\end{array}$ & $\mathrm{O}$ & 2420.45 \\
\hline Hongkong & 1 & O & O & O & 2011.4 & $\mathrm{O}$ & 2011.4 \\
\hline Haikou & 1 & $\mathrm{O}$ & 0 & $\mathrm{O}$ & 127.13 & o & 127.13 \\
\hline $\begin{array}{l}\text { Laem } \\
\text { Chabang }\end{array}$ & $\begin{array}{c}0.79 \\
1\end{array}$ & $\mathrm{O}$ & -1091.818 & -102.476 & 679.4 & 178.971 & 858.371 \\
\hline $\begin{array}{c}\text { Guangzho } \\
\mathrm{u}\end{array}$ & $\begin{array}{c}0.78 \\
1\end{array}$ & -35.004 & O & -313.193 & 1762.49 & 494.234 & 2256.724 \\
\hline Qinzhou & $\begin{array}{c}0.39 \\
7\end{array}$ & O & -984.182 & -71 & 94.2 & 143.309 & 237.509 \\
\hline Zhuhai & $\begin{array}{c}0.10 \\
3\end{array}$ & -7.953 & $\mathrm{O}$ & -43.894 & 133.77 & 1164.073 & 1297.843 \\
\hline
\end{tabular}

\subsection{Technical Efficiency and Scale Efficiency}

To calculate and analyze the ports' aggregate efficiency, pure technical efficiency and scale efficiency and obtain the results are shown in the Table 10.

The Hong Kong port has the efficiency advantage in the competition (pure technical efficiency and scale efficiency are achieved DMU efficiency).

Shenzhen port, Haikou port, Zhanjiang port and Yangpu port's pure technical efficiency value are 1, scale efficiency value is between 0.3-0.8. But besides Shenzhen port shows decreasing return to scale, the other ports exhibit increasing return to scale. It is indicated that Haikou, Zhanjiang and Yangpu ports enlarge the operational scale will have returns to scale increasing effect, and then competitiveness will be strengthened. Conversely, Shenzhen port should reduce the scale of operation. 
Laem Chabang port, Guangzhou port and Zhuhai port's pure technical efficiency value below their scale efficiency, indicating that the major cause of inefficiency is pure technical inefficiency. That is, the output is appropriate at the current level of input.

Relatively, Qinzhou port's pure technical efficiency value higher than scale efficiency value means that the major cause of inefficiency is scale efficiency. A large throughput needs the large scale to support. Qinzhou port should pay attention to the production scale.

Table 10: Technical Efficiency Value and Scale Efficiency Value

\begin{tabular}{ccccc}
\hline Port & CRS & VRS & Scale & RTS \\
\hline Hong Kong & 1 & 1 & 1 & Constant \\
Guangzhou & 0.509 & 0.512 & 0.994 & Increasing \\
Laem Chabang & 0.737 & 0.817 & 0.902 & Increasing \\
Shenzhen & 0.741 & 1 & 0.741 & Decreasing \\
Zhuhai & 0.101 & 0.160 & 0.631 & Increasing \\
Haikou & 0.619 & 1 & 0.619 & Increasing \\
Qinzhou & 0.281 & 0.596 & 0.471 & Increasing \\
Zhanjiang & 0.359 & 1 & 0.359 & Increasing \\
Yangpu & 0.163 & 1 & 0.163 & Increasing \\
\hline
\end{tabular}

Note: CRS: aggregate efficiency (technical efficiency) based on constant return to scale; VRS: pure technical efficiency based on variable return to scale; scale: scale efficiency=CRS/VRS; RTS: returns to scale (increasing, decreasing and constant) 
Table 4: Relative Efficiency Scores under Input-Oriented CCR Model

\begin{tabular}{|c|c|c|c|c|c|c|c|c|c|c|c|c|c|}
\hline Port & $\begin{array}{l}\text { Sco } \\
\text { re }\end{array}$ & \multicolumn{3}{|c|}{ Number of berths } & \multicolumn{3}{|c|}{ Berth length } & \multicolumn{3}{|c|}{ Terminal area } & \multicolumn{3}{|c|}{ Container throughput } \\
\hline $\begin{array}{l}\text { Hong } \\
\text { Kong }\end{array}$ & 1 & $\mathrm{o}$ & $\mathrm{O}$ & 24 & $\mathrm{o}$ & $\mathrm{o}$ & 7694 & o & o & 279 & o & $\mathrm{O}$ & 2011.4 \\
\hline $\begin{array}{l}\text { Shenzh } \\
\text { en }\end{array}$ & $\begin{array}{c}0.7 \\
41\end{array}$ & -10.119 & $\mathrm{o}$ & 28.881 & -4396.153 & $\begin{array}{c}- \\
3288.1 \\
51\end{array}$ & $\begin{array}{c}9258.6 \\
97\end{array}$ & -205.498 & $\begin{array}{c}- \\
250.76 \\
3\end{array}$ & $\begin{array}{c}335 \cdot 73 \\
9\end{array}$ & o & o & $\begin{array}{c}2420.4 \\
5\end{array}$ \\
\hline $\begin{array}{l}\text { Laem } \\
\text { Chaban } \\
\text { g }\end{array}$ & $\begin{array}{l}0.7 \\
37\end{array}$ & -2.893 & o & 8.107 & -1220.492 & 820.67 & $\begin{array}{c}2598.8 \\
38\end{array}$ & $-67 \cdot 515$ & -94.921 & 94.239 & o & o & 679.4 \\
\hline Haikou & $\begin{array}{c}0.61 \\
9\end{array}$ & -1.144 & -0.339 & 1.517 & -299.703 & o & $\begin{array}{c}486.29 \\
7\end{array}$ & -11.439 & -0.927 & 17.634 & $\mathrm{o}$ & $\mathrm{O}$ & 127.13 \\
\hline $\begin{array}{l}\text { Guangz } \\
\text { hou }\end{array}$ & $\begin{array}{l}0.5 \\
09\end{array}$ & $-33 \cdot 377$ & -13.593 & 21.03 & -6499.13 & o & $\begin{array}{c}6741.8 \\
7\end{array}$ & -441.682 & $\begin{array}{c}- \\
213.70 \\
4\end{array}$ & $\begin{array}{c}244.47 \\
4\end{array}$ & $\mathrm{O}$ & o & $\begin{array}{c}1762.4 \\
9\end{array}$ \\
\hline $\begin{array}{l}\text { Qinzho } \\
\mathrm{u}\end{array}$ & $\begin{array}{c}0.2 \\
81\end{array}$ & -2.876 & $\mathrm{o}$ & 1.124 & -1653.704 & $\begin{array}{c}285.96 \\
3\end{array}$ & $\begin{array}{c}360.33 \\
3\end{array}$ & -113.602 & -31.331 & 13.066 & o & o & 94.2 \\
\hline Yangpu & $\begin{array}{c}0.16 \\
3\end{array}$ & -7.535 & -1.141 & 0.324 & -1419.934 & $\begin{array}{c}- \\
172.174\end{array}$ & $\begin{array}{c}103.89 \\
2\end{array}$ & $-19 \cdot 377$ & o & 3.767 & o & o & 27.16 \\
\hline Zhuhai & $\begin{array}{l}0.1 \\
01\end{array}$ & -21.582 & -0.822 & 1.596 & $\begin{array}{c}- \\
4566.303\end{array}$ & o & 511.697 & -205.565 & -4.48 & 18.555 & o & o & 133.77 \\
\hline
\end{tabular}


Table 5: The Percentage Difference Comparison between Target and Actual Values under Input-Oriented CCRModel

\begin{tabular}{cccccccccc}
\hline DMU & Port & Shenzhen & Laem Chabang & Haikou & Guangzhou & Zhanjiang & Qinzhou & Yangpu & Zhuhai \\
\hline Input1 & Projection & 28.881 & 8.107 & 1.517 & 21.03 & 0.717 & 1.124 & 0.324 & 1.596 \\
& Origin & 39 & 11 & 3 & 68 & 2 & 4 & 9 \\
& Difference & $25.95 \%$ & $26.30 \%$ & $49.43 \%$ & $69.07 \%$ & $64.15 \%$ & $71.90 \%$ & $96.40 \%$ & $93.35 \%$ \\
\hline Input2 & Projection & 9258.697 & 2598.838 & 486.297 & 6741.87 & 229.971 & 360.333 & 103.892 & 511.697 \\
& Origin & 16943 & 4640 & 786 & 13241 & 678 & 2300 & 1696 & 5078 \\
& Difference & $45.35 \%$ & $43.99 \%$ & $38.13 \%$ & $49.08 \%$ & $66.08 \%$ & $84.33 \%$ & $93.87 \%$ & $89.92 \%$ \\
\hline Input3 & Projection & 335.739 & 94.239 & 17.634 & 244.474 & 8.339 & 13.066 & 3.767 & 18.555 \\
& Origin & 792 & 256.6757 & 30 & 899.86 & 67.8 & 158 & 23.1445 & 228.6 \\
& Difference & $57.61 \%$ & $63.28 \%$ & $41.22 \%$ & $72.83 \%$ & $87.70 \%$ & $91.73 \%$ & $83.72 \%$ & $91.88 \%$ \\
\hline
\end{tabular}

Table 7: The Percentage Difference Comparison between Target and Actual Values under Output-Oriented CCRModel

\begin{tabular}{cccccccccc}
\hline \multirow{2}{*}{ DMU } & Port & Shenzhen & $\begin{array}{c}\text { Laem } \\
\text { Chabang }\end{array}$ & Haikou & Guangzhou & Zhanjiang & Qinzhou & Yangpu & Zhuhai \\
\hline \multirow{2}{*}{ Output } & Origin & 2420.45 & 679.4 & 127.13 & 1762.49 & 60.12 & 94.2 & 27.16 & 133.77 \\
& Projection & 3268.525 & 921.892 & 205.48 & 3461.522 & 167.617 & 335.233 & 166.856 & 1327.514 \\
& Difference & $25.95 \%$ & $26.30 \%$ & $38.13 \%$ & $49.08 \%$ & $64.13 \%$ & $71.90 \%$ & $83.72 \%$ & $89.92 \%$ \\
\hline
\end{tabular}

Table8:The Relative Efficiency Results of Inefficiency Ports under Input-Oriented BCC Model

\begin{tabular}{|c|c|c|c|c|c|c|c|c|c|c|}
\hline DMU & & & Input 1 & & 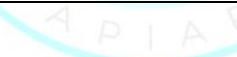 & Input 2 & & & Input 3 & \\
\hline Port & $\begin{array}{c}\text { Scor } \\
\mathrm{e}\end{array}$ & $\begin{array}{l}\text { Proportionat } \\
\text { e movement }\end{array}$ & $\begin{array}{c}\text { Slack } \\
\text { movemen } \\
\mathrm{t}\end{array}$ & $\begin{array}{c}\text { Projectio } \\
\mathrm{n}\end{array}$ & $\begin{array}{l}\text { Proportionat } \\
\text { e movement }\end{array}$ & $\begin{array}{c}\text { Slack } \\
\text { movemen } \\
\mathrm{t} \\
\end{array}$ & $\begin{array}{l}\text { Projectio } \\
\mathrm{n}\end{array}$ & $\begin{array}{l}\text { Proportionat } \\
\text { e movement }\end{array}$ & $\begin{array}{c}\text { Slack } \\
\text { movemen } \\
\mathrm{t}\end{array}$ & Projection \\
\hline Laem & 0.81 & -2.018 & 0 & 8.982 & -851.159 & -884.165 & 2904.676 & -47.084 & -74.762 & 134.829 \\
\hline Chabang & 7 & & & & & & & & & \\
\hline Qinzhou & $\begin{array}{c}0.59 \\
6\end{array}$ & -1.616 & $\mathrm{O}$ & 0.384 & -929.062 & -570.4 & 800.538 & -63.823 & -22.689 & 71.489 \\
\hline Guangzhou & $\begin{array}{c}0.51 \\
2\end{array}$ & -33.173 & -13.601 & 21.226 & -6459.539 & $\mathrm{O}$ & 6781.461 & -438.991 & -214.762 & 246.107 \\
\hline Zhuhai & 0.16 & -20.17 & -0.756 & 3.074 & -4267.657 & 0 & 810.343 & -192.12 & -5.602 & 30.877 \\
\hline
\end{tabular}


4.6. Super Efficiency

Table11: Super Efficiency Model Results

\begin{tabular}{ccccc}
\hline Port & VRS-SE value & Ranking & CRS-SE value & Ranking \\
\hline Shenzhen & Big & 1 & 0.7405 & 2 \\
Hong Kong & 2.3516 & 2 & 1.8427 & 1 \\
Haikou & 1.9351 & 3 & 0.6187 & 4 \\
Zhanjiang & 1.5 & 4 & 0.3587 & 6 \\
Yangpu & 1.2962 & 5 & 0.1628 & 8 \\
Laem Chabang & 0.8166 & 6 & 0.7370 & 3 \\
Qinzhou & 0.5961 & 7 & 0.2810 & 7 \\
Guangzhou & 0.5122 & 8 & 0.5092 & 5 \\
Zhuhai & 0.1596 & 9 & 0.1008 & 9
\end{tabular}

Note: VRS-SE value: super efficiency value from a variable return to scale; CRS-SE value: super efficiency value from a constant return to scale

With respect to the relative efficiency ranking, the $\mathrm{BCC}$ model has 5 efficiency ports, but it is not possible to differentiate the five ports since the ports efficiency score is 1 . Therefore, it is necessary to use super efficiency model to rank the ports.

The ports ranking based on constant returns to scale and variable returns to scale are shown in Table 11. Shenzhen port's super efficiency value shows as Big based on variable returns to scale. It means that Shenzhen port is DEA efficiency, whether to increase input or decrease output. However, it is relative in the sample ports.

The ranking under variable returns to scale has changed. Shenzhen port ranks first, followed by Hong Kong port. Zhanjiang port move up two places to No 4 and Yangpu port move 3 places to No 5 respectively, shows the strong competitiveness. On the contrary, Laem Chabang port and Guangzhou port dropped 3 places to No 6 and No 8 respectively. Qinzhou port ranking does not change. Zhuhai port is ranked in the bottom shows the weak competitiveness. High concentration of super efficiency value illustrates the intense competition level of ports of PBGEC.

\section{Conclusion}

In this paper, the relative efficiency and competitive position of Qinhzou port, the maritime route between Thailand and China, are researched through the comparison with the rest 8 ports under PBGEC. The conclusion could be presented thereafter. The results of input and output oriented CCR model show that Qinzhou port efficiency score is 0.281 , and the results of input and output oriented BCC model indicate that Qinzhou port efficiency scores are 0.596 and 0.397 respectively, both of which reveal that Qinzhou port exists input redundancy and insufficient output in the sample ports. In addition, the pure technical efficiency and technical efficiency results also imply that the main cause of Qinzhou port inefficiency relies on scale efficiency. Moreover, the ranking of the super efficiency model indicates that Qinzhou port performs with weak competitiveness.

There are some limitations of this study in following aspects. Firstly, due to the data of sample ports were difficult to obtain, this study just consider the cross-sectional data of year 2015. Secondly, as DEA analysis calculates the relative efficiency based on the selected samples, in this study we concentrate on the 9 container ports of PBGEC for the year 2015, so the DEA results probably would be different if the sample ports were different or new data of another year are included. 
And the implication for further study could be summarized. Upcoming research should apply panel data to evaluate the efficiency of the sample ports with larger sample ports and variables. And various research objects should be widely applied. Additionally, container terminals comparison between two or more countries also should be applied to further research. Further, for the feasibility of this research for the policy makers, how to enhance the overall competitiveness of Qinzhou port should be taken in no time. 


\section{References}

i. Cruz, M. R. P. D., 2012. Competitiveness and Strategic Positioning of Seaports: the Case of Iberian Seaports, s.l.: Universidade da Beira Interior.

ii. Cullinane, K. \& Wang, T., 2010. The Efficiency Analysis of Container Port Production Using DEA Panel Data Approaches. OR spectrum, 32(3), pp. 717-738.

iii. Dyck, G. K. v. \& Ismael, H. M., 2015. Multi-Criteria Evaluation of Port Competitiveness in West Africa Using Analytic Hierarchy Process (AHP). American Journal of Industrial and Business Management, 5(6), p. 432.

iv. Golany, B. \& Roll, Y., 1989. An Application Procedure for DEA. Omega, 17(3), pp. 237-250.

v. Grosso, M. \& Monteiro, F., 2009. Relevant Strategic Criteria When Choosing a Container Port the Case of the Port of Genoa, s.l.: Research in Transport and Logistics.

vi. Hosokawa, D., 2009. Pan-Beibu Gulf Economic Cooperation. China's New Initiative in Cooperation with ASEAN, 6o(2), pp. 67-78.

vii. J. Tongzon, J. \& Heng, 2005. Port Privatization, Efficiency and Competitiveness: Some Empirical Evidence From Container Ports (terminals). Transportation Research Part A Policy and Practice, 39(5), pp. 405-424.

viii. Jafari, H., Noshadi, E. \& Khosheghbal, B., 2013. Ranking Ports Based on Competitive Indicators by Using ORESTE Method. International Research Journal of Applied and Basic Science, 4(6), pp. 1492-1498.

ix. $\quad$ Norman, M. \& Stoker, B., 1991. Data Envelopment Analysis:The Assessment of Performance. 1st ed. New York: John Wiley \& Sons.

x. Polyzos, S. \& Niavis, S., 2013. Evaluating Port Efficiency in the Mediterranean. International Journal of Data Analysis Techniques and Strategies, 5(1), pp. 84-100.

xi. Qinzhou Municipal Bureau of Statistics, 2016. Statistical Communiqué of Qinzhou on the 2015 National Economic and Social Development. [Online] Available at: http://www.qinzhou.gov.cn [Accessed 2 December 2016].

xii. Rudjanakanoknad, J., Suksirivoraboot, W. \& Sukdanont, S., 2014. Evaluation of International Ports in Thailand through Trade Facilitation Indices from Freight Forwarders. Procedia Social and Behavioral Sciences, Volume 111, pp. 1073-1082.

xiii. Song, D. W. \& Yeo, K. T., 2004. A Competitive Analysis of Chinese Container Ports Using the Analytic Hierarchy Process. Maritime Economics \& Logistics, 6(1), pp. 34-52.

xiv. Tongzon, J., 2001. Efficiency Measurement of Selected Australian andother International Ports Using Data Envelopment Analysis. Transportation Research Part A: Policy and Practice, 35(2), pp. 107-122.

xv. Voorde, E. V. d. \& Winkelmans, W., 2002. A General Introduction to Port Competition and management. Port Competitiveness, s.l.: An economic and legal analysis of the factors determining the competitiveness of seaports.

xvi. Vuković, D., Jovanović, A. \& Đukić, M., 2012. Defining competitiveness through the theories of New Economic Geography and Regional Economy. Journal of the Geographical Institute, 62(3), pp. 49-64.

xvii. Yeo, G. T., Roe, M. \& Dinwoodie, J., 2008. Evaluating the Competitivenessof Container Ports in Korea and China. Policy and Practice, 42(6), pp. 910-921.

xviii. Yeo, K. T. \& Song, D. W., 2003. An Evaluation of Container Ports in China and Korea with the Analytic Hierarchy Process. Journal of the Eastern Asia Society for Transportation studies, 5(3), pp. 726-741.

xix. Yuen, C. L. A., Zhang, A. \& Cheung, W., 2012. Port Competitiveness from the Users' Perspective: An analysis of major container ports in China and its neighboring countries.. Research in Transportation Economics, 35(1), pp. 34-40. 